\title{
Paraprobiotics and postbiotics: Contemporary and promising natural antibiotics alternatives and their applications in the poultry field
}

\author{
Wafaa A. Abd El-Ghany* \\ Poultry Diseases Department, Faculty of Veterinary Medicine, Cairo University, Giza, Egypt
}

\begin{abstract}
With the high rise of drug resistance in microbial populations, there has been a surge in researches to find new natural antibiotics alternative compounds that can be used safely in both humans and animals. The main goals of using this category of alternatives are maintaining the gut microbiome in healthy conditions and preventing the attachment of pathogenic organisms at the early life stages. Probiotics, prebiotics, and synbiotics have been widely used for several years as growth promoters and as preventive measures against several enteric pathogens with successful results. Recently, paraprobiotics and postbiotics are derivatives of probiotic cultures and have been used in humans, animals, and poultry. They are regarded as immunostimulators, anti-inflammatory, antioxidants, and anti-microbial, as well as growth promoters. Till now, there is scanty information about the use of paraprobiotics and postbiotics in animals or in the poultry sector. Accordingly, this review article has focused on defining these new categories of natural alternatives with descriptions of their types, functions, and uses, especially in the poultry field.
\end{abstract}

Keywords: Immunomodulation, Paraprobiotics, Performance, Postbiotics, Poultry.

\section{Introduction}

Due to the rapid increase in human population worldwide, there is a continuous demand for animal protein. Poultry meat is considered as one of the most important, available, and valuable sources of animal protein (Zuidhof et al., 2014). A lot of diseases can affect the poultry industry, causing severe economic losses. Antibiotics are used to prevent such diseases and are also used as growth promoters and feed additives to improve the growth performance parameters (Durso and Cook, 2014). Unfortunately, prolonged, indiscriminate, and extensive administration of antibiotics in the poultry field have raised concerns relating to residues in meat and egg products (Shareef et al., 2009), development of antibiotic-resistant bacterial populations (Shazali et al., 2014), and consequently, there is an adverse effect on the health of poultry and humans (Ajuwon, 2015). Therefore, many countries have banned the administration of antibiotics in the feed. In 2017, the Federal Government imposed the Food and Drug Administration Veterinary Feed Directive to reduce the use of antibiotics as growth promoters. Nowadays, the biggest challenge facing producers of poultry is how to increase the production parallel with ensuring the safety and health of the product. In view of the prohibiting antibiotics use as growth promoters, several natural alternatives have recently gained attention, they were also encouraged and experimented for production of safe and foodborne disease-free products. Paraprobiotics and postbiotics are natural antibiotics alternatives.
There is still a dearth of information regarding the use of paraprobiotics and postbiotics for improving the health and maintaining the immune status of the host, as well as preventing diseases conditions. Hence, the objectives of this review article were to give more attention on paraprobiotics and postbiotics regarding their definitions, types, functions, and their uses, especially in the poultry field.

\section{Types of natural antibiotic alternatives}

Microbiota inhibiting the avian gut have an important role in the nutritional, immunological, and physiological status of the host as their compositions and activities are affected by feed (Rehman et al., 2007). These microbiota are adversely affected by the prolonged use of antibiotics. "Biotic" feed additives, probiotics, prebiotics, synbiotics, paraprobiotics, and postbiotics, are some of the promising antibiotics alternatives. They have been used to alleviate the problems of using antibiotics, improve both the production and the intestinal health, as well as control enteric pathogens (Jahromi et al., 2016).

Probiotics are sufficient numbers of defined and viable live bacterial or fungal cultures that have beneficial health functions in the host (Fuller, 1989). Probiotics can induce many obvious functions through alteration of gut microflora, colonization in specific intestinal niches (Howarth and Wang, 2013), and modulation of both cell-mediated and humoral immune responses (Shang et al., 2008). Although prebiotics are indigestible feed ingredients by animals, they can enhance the growth of specific beneficial microbial population numbers and 
inhibit the pathogenic ones (Alloui et al., 2013). Some researchers have described prebiotics as nutraceuticals or functional foods (Roberfroid, 2007). It was found that prebiotics could control the growth of some pa thogens, like Salmonellae and Escherichia coli (E. coli), stimulate the growth of beneficial Lactobacilli and Bifidobacteria, and accordingly improve the health and performance of animals (Bogusławska-Tryk et al., 2012). Synbiotics are combinations of probiotics and prebiotics in which prebiotic selectively favors the probiotic (Schrezenmeir and De Vrese, 2001).

Despite numerous probiotics' notable beneficial effects, inconsistent results demonstrate that probiotics need to be more specialized for animal species and their overall efficacy is debated. Bacterial organisms of some probiotics show and acquire antibiotic-resistance genes encoded by plasmids that transfer between microorganisms (Gueimonde et al., 2013). Moreover, it was detected that probiotics can have a negative impact on the host by inducing local inflammation in healthy hosts and increasing the severity of tissue inflammation in the hosts with inflammatory bowel disease (IBD) (Tsilingiri et al., 2012). Subsequently, in the near future, probiotics might be completely substituted with paraprobiotics and postbiotics.

\section{Definition of paraprobiotics}

Probiotics contain both living and dead cells with a low shelf life (Lahtinen, 2012). It was found that the dead cells of probiotics could be fractionated and their metabolites showed a wide range of bioactivities (Adams, 2010). Inactivation of probiotics occurs by heat, chemicals such as formalin, radiation with gamma or ultraviolet rays, and sonication (Zorzela et al., 2017). The inactivation process may affect the structural composition of the cells and may influence their immunomodulatory activities (Taverniti and Guglielmetti, 2011). Accordingly; Taverniti and Guglielmetti (2011) proposed a term paraprobiotic (ghost probiotics) to define the non-viable, intact, or broken microbial cells or crude cell extracts (complex chemical composition), which, when taken in adequate amounts either through oral or topical routes, provide benefit to the human or animal consumer.

\section{Paraprobiotics cultures}

Different species of bacterial cultures were used as paraprobiotics. The most common paraprobiotic species are Lactobacilli and Bifidobacterium as they proved their efficacy after inactivation, especially with heat.

\section{Function of paraprobiotics}

Immunomodulation and anti-inflammatory

Paraprobiotics were found to maintain their immunomodulatory capacity beyond their viability (Taverniti and Guglielmetti, 2011). It was suggested that the killed probiotics may be as effective as the live ones in modulating inflammatory stimulation, after experiments in young rodent (Lopez et al., 2008). Dead probiotic bacteria showed different biological responses in the stimulation of gut immune system, antiinflammatory effects, reduction of interleukin (IL)-8 production, and stimulation of IL-6 production (Adams, 2010). Heat inactivation of Lactobacillus casei) and Lactobacillus fermentum revealed a modulation of inflammatory responses through regulating IL-10, B-defensin, and other pro-inflammatory cytokines, while inactivation of Bifidobacterium breve and Bifidobacterium bifidum showed an increase in the secretion of IL-10, which is an anti-inflammatory cytokine (Habil et al., 2014). Guglielmetti et al. (2008) proved that dead probiotics have an adhesive cell surface lipoprotein, which was detected in strains of $B$. bifidum and anti-inflammatory substances in Lactobacillus acidophilus and Lactobacillus plantarum. Inactivated preparations of cultures containing Lactobacillus rhamnosus, L. plantarum, L. acidophilus, Lactobacillus helveticus, L. casei, B. breve, B. bifidum, and $E$. coli induced effective immune responses (Taverniti and Guglielmetti, 2011). Heatkilled L. rhamnosus decreased lipopolysaccharides, induced pro-inflammatory mediators, as well as increased anti-inflammatory mediators in gastrostomyfed young rats (Li et al., 2009). Several inactivated and non-viable probiotic cultures were found to be immune potentiators through different mechanisms, including increasing salivary gene expression (Kotani et al., 2010), modulation of T-cell responses (Hirose et al., 2006), and stimulation of IgA production (Konstantinov et al., 2008). The immunomodulatory effects of heat-inactivated $L$. plantarum strains were tested in humans and pigs and the results revealed that his type of paraprobiotic increased the innate and acquired immune responses due to the production of interferons type 1 and IL-12 (Hirose et al., 2006). In a gnotobiotic mouse model, heat inactivation of $B$. breve helped in immunemodulation and regulation which suppressed the production of pro-inflammatory cytokine production in spleen cells and affected the gene expression, as well as intestinal metabolism (Sugahara et al., 2017).

\section{Antimicrobial and maintaining of gut health}

Heat-inactivated Lactobacilli were found in the intestinal lumen (Maldonado Galdeano and Perdigon, 2004), which enhanced the gut epithelial barrier (Montalto et al., 2004) and inhibited the pathogen adhesion to the gut mucosa through competitive exclusion (Ouwehand et al., 2000). In newly hatched chicks, killed strains of Enterococcus faecalis prevented vancomycin-resistant enterococcus intestinal colonization (Sakai et al., 2006). In patients with viral hepatitis $C$, paraprobiotic of $E$. faecalis culture reduced alanine aminotransferase levels due to the change in microbiota (Oo et al., 2016). Inactive Saccharomyces boulardii shortened the duration of diarrhea in acute rotavirus gastroenteritis children after oral administration (Grandy et al., 2010). Bifidobacterium lactis treatment shortened the diarrheal period in acute rotavirus gastroenteritis in 5 months to 
5 -year-old children after oral rehydration (Erdogan et al., 2012).

\section{Definition of postbiotics}

Postbiotics are soluble non-viable metabolites produced by a bacterial or probiotic metabolic process (Tsilingiri et al., 2012; Klemashevich et al., 2014). So, postbiotics shared probiotics with the same mode of action but differed being non-living organisms (Thanh et al., 2009). Moreover, postbiotics are fed products such as organic acids and bacteriocins that are produced by beneficial intestinal microbiota and are able to reduce the gut $\mathrm{pH}$, prohibit opportunistic pathogens proliferation, and consequently have a positive influence on host health (Aguilar-Toalá et al., 2018). Bacteriocins, shortchain fatty acids, peptides, and proteins are considered as postbiotics inhibitory metabolites (Cicenia et al., 2014). Postbiotics have been classified as promising alternatives to antimicrobials due to their multiple health benefits.

\section{Postbiotics producers}

The cell wall components and cytoplasmic extracts of various Lactobacilli species, including $L$. acidophilus, L. casei, L. fermentum, L. rhamnosus, Lactobacillus paracasei, Lactobacillus delbrueckil subsp. Bulgaricus, Lactobacillus gasseri, L. helveticus, Lactobacillus reuteri, and Lactobacillus johnsonni, were found to be highly effective postbiotics (Vidal et al., 2002; Matsuguchi et al., 2003; Choi et al., 2006; Kim et al., 2011; Cicenia et al., 2016; TiptiriKourpeti et al., 2016; Johnson et al., 2019). In addition, Bifidobacterium species (Tejada-Simon and Pestka, 1999), Faecalibacterium prausnitzii (Sokol et al., 2008), and Bacillus coagulans (Jensen et al., 2010) showed postbiotics properties. Strains of $L$. plantarum, either alone or in combination, are the most common postbiotics producers (Thanh et al., 2009; Thu et al., 2011). The strains of L. plantarum demonstrated efficiency in rats (Loh et al., 2009), pigs (Loh et al., 2013), broiler chickens (Thanh et al., 2009; Loh et al., 2010; Rosyidah et al., 2011; Jahromi et al., 2016; Kareem et al., 2016, 2017), layer chickens (Loh et al., 2014), and ruminants (Izuddin et al., 2019), as well as humans (Kim et al., 2011). Saccharomyces cerevisiae is also a yeast species that has been used for the production of metabolites after anaerobic fermentation in a proprietary medium and drying of the liquid (Jensen et al., 2007).

\section{Functions of postbiotics}

Immunomodulation and anti-inflammatory

Postbiotics have exhibited immunostimulation properties. Both cell-mediated and humoral immune responses have been activated. The cell wall components and cytoplasmic extracts of Bifidobacterium sp. and Lactobacilli, including $L$. acidophilus, L. casei, L. delbrueckil subsp. Bulgaricus, L. gasseri, and L. helveticus, showed ability to stimulate immune cells and produced cytokines and nitric oxide in vitro (Tejada-Simon and Pestka, 1999). Moreover,
Lee et al. (2002) found that the purified cell wall supernatants and cell-free extracts of Bifidobacterium provoked the rapid activation of macrophages through the formation of actin filament and development of vacuole and cytokines production. The modulation of pro-inflammatory responses of the host by lipoteichoic acids of probiotics bacteria as $L$. casei and $L$. fermentum was proved (Matsuguchi et al., 2003). It was detected that $F$. prausnitzii exhibited anti-inflammatory effects on cellular and trinitrobenzenesulfonic acid colitis models as a result of secreted metabolites that block NF-kappa B activation and IL-8 production (Sokol et al., 2008). Jensen et al. (2010) suggested that the cell wall components and metabolites of $B$. coagulans can possess strong immune modulating effects in vitro; in addition, the anti-inflammatory effects and production of IL-10 are important in the treatment of IBD, as well as in enhancing healthy immune system. In this regard, Tsilingiri et al. (2012) showed that the cell-free supernatants of $L$. paracasei could protect against the inflammatory activities of invasive Salmonella in healthy tissues, as well as in lowering ongoing inflammatory processes in IBD tissues. Also, Kim et al. (2011) demonstrated that the lipoteichoic acids of $L$. plantarum attenuated the pro-inflammatory signaling induced by Shigella flexneri in human monocytic THP-1 cells, which showed by a significant reduction in tumor necrotizing factor (TNF)- $\alpha$ and IL$1 \beta$ production. In the same context, feeding of lambs with metabolites of $L$. plantarum induced an increase in the level of IL- 6 mRNA and decrease in IL-1 $\beta$, IL-10, and TNF mRNA (Izuddin et al., 2019). The sonicated cell suspensions of $L$. casei proved inhibitory activity against colon carcinoma cells in mice and anti-proliferative and apoptotic cell death in cell lines (Tiptiri-Kourpeti et al., 2016). Cicenia et al. (2016) found that the postbiotic mediators of L. rhamnosus protected colonic human smooth muscle cells from lipopolysaccharides, which induced myogenic damage. Recently, other types of bacterial species were used for production of postbiotics. In broiler chickens, combinations of insulin and postbiotics revealed an upregulation of mRNA expression of IL-6 and reduction of expression of IFN and lipopolysaccharide-induced tumor necrosis factor-alpha factor gene (Kareem et al., 2017). Cultures of Pediococcus acidilactici, L. reuteri, Enterococcus faecium, and L. acidophilus were tested in broiler chickens and demonstrated great efficiency in improving the innate immunity if used alone; moreover, they inhibited the activation of immune response if used with pathogenic Clostridium perfringens (Johnson et al., 2019).

\section{Antioxidant}

The in vitro antioxidant effects of intracellular contents of Streptococcus salivarius subsp. Thermophillus and L. delbrueckii subsps. Bulgaricus (Ou et al., 2006), as well as L. acidophilus, L. jonsonnii, and L. brevis were studied (Ji et al., 2015). The results demonstrated 
that these cultures inhibited lipid peroxidation and showed resistance to hydrogen peroxide and hydroxyl radical scavenging activity and glutathione peroxidase activity. Postbiotics exhibited antioxidant effects due to an increase in the content of uronic acid which has an antioxidant effect as well as the chelating capability of ferrous ion which is involved in the formation of free radicals. Moreover, the antioxidant action of postbiotics may be related to the production of antioxidant enzymes as glutathione peroxidase, superoxide dismutase, and nicotinamide adenine dinucleotide oxidase and peroxidase. Choi et al. (2006) detected that the soluble intracellular polysaccharide fraction contents of $L$. acidophilus, L. brevis, L. casei, and L. rhamnosus could be considered as anticancer substance due to selectivity for human cancer cells and antioxidative effect in the food industry. The antioxidant activity of $S$. cerevisiae metabolites was demonstrated in human leukocytes (Possemiers et al., 2013). In poultry, L. plantarum producing postbiotics showed antioxidative activity under heat stress (Humam et al., 2019).

\section{Anti-proliferative}

Postbiotics showed anti-proliferative activity against cancer cells via regulation of immune response. The sonicated cell suspensions of $L$. casei proved inhibitory activity against colon carcinoma cells in mice and anti-proliferative and apoptotic cell death in cell lines (Tiptiri-Kourpeti et al., 2016). These types of bacteria produce some inhibitory components like proteins, nucleic acids, or lipopolysaccharides, which reduce the activity of metalloproteinase- 9 activity and consequently inhibit the cancer colon. The postbiotic mediators of L. rhamnosus protected colonic human smooth muscle cells from lipopolysaccharides which induced myogenic damage (Cicenia et al., 2016). Some postbiotics produce fatty acids such as butyrate, propionate, and acetate, which are very important for homeostasis of energy balance, cholesterol, and glucose, as well as regulation of lipid metabolism.

\section{Antibacterial}

It was demonstrated that lipoteichoic acids from $L$. johnsonni and L. acidophilus were able to maintain gut homeostasis and were used as a treatment of diseases mediated by Gram-negative bacteria or their components (Vidal et al., 2002). Postbiotics metabolites of L. plantarum with prebiotic (inulin) showed great inhibitory effect of different bacterial pathogens such as E. coli, Salmonella typhymurium, vancomycin-resistant enterococci, and Listeria monocytogens (Kareem et al., 2014). Supernatants of $L$. paracasei cells protected human healthy tissues from invasive Salmonella and downgrading the ongoing inflammatory processes in tissues with IBD (Tsilingiri et al., 2012). Postbiotic mediators of $L$. rhamnosus that were collected at different growth stages protected colonic mucosal cells from Gram-negative bacteria (Cicenia et al., 2016). The fermentation products of $S$. cerevisiae cultures clearly showed promising successful results regarding the increase in the animal's growth rate, as well as the decrease in the intestinal colonization of pathogens. It was proved that $S$. cerevisiae fermentation metabolites reduced the colonization of both $S$. typhimurium and Campylobacter coli in pigs and broiler chickens (Price et al., 2010; Hofacre et al., 2015; Feye et al., 2016; Rubinelli et al., 2016). Johnson et al. (2019) concluded that water treatment of broilers with postbiotics metabolites of $P$. acidilactici, L. reuteri, E. faecium, and $L$. acidophilus in the presence of pathogenic C. perfringens provoked the activation of immune responses to this pathogen.

\section{Intestinal health and performance parameters}

Postbiotics maintain the gut health, improve the intestinal mucosal barriers integrity, and enhance the inflammatory mediator secretion. It was found that the addition of postbiotics to broiler rations improved the intestinal villi, increased production of lactic acid bacteria, and reduced Enterobacteriaceae and fecal $\mathrm{pH}$, resulting in increased growth performance parameters and enhanced immune response and gut health (Thanh et al., 2009, Loh et al., 2010; Rosyidah et al., 2011; Kareem et al., 2017). In lambs fed postbiotics produced from L. plantarum, there were improvements in the height and width of rumen papillae and improvement in the integrity of the intestinal barrier (Izuddin et al., 2019). In piglets, liquid metabolite combinations produced by $L$. plantarum helped in increasing the growth performance parameters, including birth weight, overall weight gain, and average weight gain per day, as well as reduction in diarrhea production (Loh et al., 2013). Similarly, in layers, these metabolites contributed to improving egg quality and reduction in plasma and yolk cholesterol (Loh et al., 2014). Kareem et al. (2016) demonstrated that the addition of a combination of postbiotics and prebiotic containing inulin in the ration of broiler chickens improved the total body weight and the feed efficiency, and maintained the intestinal mucosal structure, growth factor 1, and growth hormone receptor mRNA expressions. Feeding of broiler chickens with postbiotics produced by L. plantarum indicated improvement of growth performance, intestinal morphology, and maintained gut microbiota, even under heat stress conditions (Humam et al., 2019).

\section{Conclusion}

The overall numerous health benefits arising from the use of paraprobiotics and postbiotics in humans, animals, and poultry can be classified as favorable alternatives to antibiotics. As the types and exact mechanisms of paraprobiotics and postbiotics are not fully elucidated, further research work and additional information are necessary to discover other cultures' sources and understand the characteristics and mode of actions especially in the field of poultry. 


\section{References}

Adams, C.A. 2010. The probiotic paradox: Live and dead cells are biological response modifiers. Nutr. Res. Rev. 23, 37-46.

Aguilar-Toalá, J., Garcia-Varela, R., Garcia, H., MataHaro, V., González-Córdova, A., Vallejo-Cordoba, B. and Hernández-Mendoza, A. 2018. Postbiotics: an evolving term within the functional foods field. Trends Food Sci. Technol. 75, 105-114.

Ajuwon, K.M. 2015. Toward a better understanding of mechanisms of probiotics and prebiotics action in poultry species. J. Appl. Poult. Res. 25(2), 277-283.

Alloui, M.N., Szczurek, W. and Swiatkiewicz, S., 2013. The usefulness of prebiotics and probiotics in modern poultry nutrition: A review. Ann. Anim. Sci. 13, 17-32.

Bogusławska-Tryk, M., Piotrowska, A. and burlikowska, K. 2012. Dietary fructans and their potential beneficial influence on health and performance parameters in broiler chickens. J. Cent. Europ. Agri. 13(2), 272-291.

Choi, S.S., Kim, Y., Han, K.S., You, S., Oh, S. and Kim, S.H. 2006. Effects of Lactobacillus strainson cancer cell proliferation and oxidative stress in vitro. Lett. Appl. Microbiol. 42(5), 452-458.

Cicenia, A., Santangelo, F., Gambardella, L., Pallotta, L., Iebba, V., Scirocco, A., Marignani, M., Tellan, G., Carabotti, M., Corazziari, E.S., Schippa, S. and Severi, C. 2016. Protective role of postbiotic mediators secreted by Lactobacillus Rhamnosus GG versus lipopolysaccharide-induced damage in human colonic smooth muscle cells. J. Clin. Gastroenterol. 50 (Suppl. 2), Proceedings from the 8th Probiotics, Prebiotics \& New Foods for Microbiota and Human Health meeting held in Rome, Italy on September 13-15, 2015: S140S144.

Cicenia, A., Scirocco, A., Carabotti, M., Pallotta, L., Marignani, M. and Severi, C. 2014. Postbiotic activities of Lactobacilli-derived factors. J. Clin. Gastroent. 48, S18.

Durso, L.M. and Cook, K.L. 2014. Impacts of antibiotic use in agriculture: what are the benefitsand risks? Curr. Opin. Microbiol. 19, 37-44.

Erdogan, O., Tanyeri, B., Torun, E., Gonullu, E., Arslan, H., Erenberk, U. and Oktem, F. 2012. The comparition of the efficacy of two different probiotics in rotavirus gastroenteritis in children. $\mathrm{J}$. Trop. Med. 2012, 787240.

Feye, K.M., Anderson, K.L., Scott, M.F., Mcintyre, D.R. and Carlson, S.A. 2016. Inhibition of the virulence, antibiotic resistance, and fecal shedding of multiple antibiotic-resistant Salmonella Typhimurium in broilers fed original XPCTM. Poult. Sci. 95(12), 2902-2910.

Fuller, R. 1989. Probiotics in man and animals. J. Appl. Bacteriol. 66, 365-378.
Grandy, G., Medina, M., Soria, R., Teran, C.G. and Araya, M. 2010. Probiotics in the treatment of acute rotavirus diarrhoea. A randomized, doubleblind, controlled trial using two different probiotic preparations in Bolivian children. BMC Infect. Dis. $10,253$.

Gueimonde, M., Sánchez, B., de los Reyes-Gavilán, C.G. and Margolles, A. 2013. Antibiotic resistance in probiotic bacteria. Front. Microbiol. 4, 202.

Guglielmetti, S., Tamagnini, I., Mora, D., Minuzzo, M., Scarafoni, A., Arioli, S., Hellman, J., Karp, M. and Parini, C. 2008. Implication of an outer surface lipoprotein in adhesion of Bifidobacterium bifidum to Caco-2 cells. Appl. Environ. Microbiol. 74, 4695-4702.

Habil, N., Abate, W., Beal, J. and Foey, A.D. 2014. Heat-killed probiotic bacteria differentially regulate colonic epithelial cell production of human $\beta$-defensin-2: Dependence on inflammatory cytokines. Benef. Microbes 5, 483-495.

Hirose, Y., Murosaki, S., Yamamoto, Y., Yoshikai, Y. and Tsuru, T. 2006. Daily intake of heat-killed Lactobacillus plantarum L-137 augments acquired immunity in healthy adults. J. Nutr. 136, 30693073.

Hofacre, C.L., Mathis, G.F., Mcintyre, D.R. and Broomhead, J.P. 2015. Effects of original XPC on cecal colonization by Campylobacter coli in broiler chickens: preliminary report. PO 225, 1-8.

Howarth, G.S. and Wang, H. 2013. Role of endogenous microbiota, probiotics and their biological products in human health. Nutrients 5, 58-81.

Humam, A.M., Loh, T.C., Foo, H.L., Samsudin, A.A., Mustapha, N.M., Zulkifli, I. and Izuddin, W.I. 2019. Effects of feeding different postbiotics produced by Lactobacillus plantarum on growth performance, carcass yield, intestinal morphology, gut microbiota composition, immune status, and growth gene expression in broilers under heat stress. Anim. (Basel) 9(9), 644.

Izuddin, W.I., Loh, T.C., Foo, H.L., Samsudin, A.A. and Humam, A.M. 2019. Postbiotic Lactobacillus plantarum RG14 improves ruminal epithelium growth, immune status and upregulates the intestinal barrier function in post-weaning lambs. Sci. Rep. 9, 9938.

Jahromi, M.F., Altaher, Y.W., Shokryazdan, P., Ebrahimi, R., Ebrahimi, M., Idrus, Z., Tufarelli, V. and Liang, J.B. 2016. Dietary supplementation of a mixture of Lactobacillus strains enhances performance of broiler chickens raised under heat stress conditions. Int. J. Biometeorol. 60, 10991110.

Jensen, G.S., Benson, K.F., Carter, S.G. and Endres, J.R. 2010. Ganden BC ${ }^{30 \mathrm{TM}}$ cell wall and metabolites: anti-inflammatory and immune modulating effects in vitro. BMC Immunol. 11, 1-15. 
Jensen, G.S., Hart, A.N. and Schauss, A.G. 2007. An anti-inflammatory immunogen fromyeast culture induces activation and alters chemokine receptor expression on human natural killer cells and B lymphocytes in vitro. Nutr. Res. 27, 327-335.

Ji, K., Jang, N.Y. and Kim, Y.T. 2015. Isolation of lactic acid bacteria showing antioxidative andprobiotic activities from kimchi and infant feces. J. Microbiol. Biotechnol. 25, 1568-1577.

Johnson, C.N., Kogut, M.H., Genovese, K., He, H., Kazemi, S. and Arsenault, R.J. 2019. Administration of a postbiotic causes immunomodulatory responses in broiler gut and reduces disease pathogenesis following challenge. Microorganisms 7(8), 268.

Kareem, K.Y., Ling, F.H., Chwen, L.T., Foong, O.M. and Asmara, S.A. 2014. Inhibitory activity of postbiotic produced by strains of Lactobacillus plantarum using reconstituted media supplemented with inulin. Gut Pathog. 6, 23.

Kareem, K.Y., Loh, T.C., Foo, H.L., Akit, H. and Samsudin, A.A. 2016. Effects of dietary postbiotic and inulin on growth performance, IGF1 and GHR mRNA expression, faecal microbiota and volatile fatty acids in broilers. BMC Vet. Res. 12, 163.

Kareem, K.Y., Loh, T.C., Foo, H.L., Asmara, S.A. and Akit, H. 2017. Influence of postbiotic RG14 and inulin combination on cecal microbiota, organic acid concentration, and cytokine expression in broiler chickens. Poult. Sci. 96, 966-975.

Kim, H.G., Lee, S.Y., Kim, N.R., Lee, H.Y., Ko, M.Y., Jung, B.J., Kim, C.M., Lee, J.M., Park, J.H., Han, S.H. and Chung, D.K. 2011. Lactobacillus Plantarum lipoteichoic acid down-regulated Shigella Flexneri peptidoglycan-induced inflammation. Mol. Immunol. 48(4), 382-391.

Klemashevich, C., Wu, C., Howsmon, D., Alaniz, R.C., Lee, K., Jayaraman, A. 2014. Rational identification of diet-derived postbiotics for improving intestinal microbiota function. Curr. Opin. Biotech. 26, 8590.

Konstantinov, S.R., Smidt, H., de Vos, W.M., Bruijns, S.C., Singh, S.K., Valence, F., Molle, D., Lortal, S., Altermann, E., Klaenhammer, T.R. and Kooyk, Y. 2008. S layer protein a of Lactobacillus acidophilus NCFM regulates immature dendritic cell and T cell functions. Proc. Natl. Acad. Sci. U. S. A. 105(49), 19474-19479.

Kotani, Y., Shinkai, S., Okamatsu, H., Toba, M., Ogawa, K., Yoshida, H., Fukaya, T., Fujiwara, Y., Chaves, P.H., Kakumoto, K. and Kohda, N. 2010. Oral intake of Lactobacillus pentosus strain b240 accelerates salivary immunoglobulin a secretion in the elderly: a randomized, placebo-controlled, double-blind trial. Immun. Ageing 7, 11.

Lahtinen, S. 2012. Probiotic viability-does it matter? Microb. Ecol. Health Dis. 23, 18567.
Lee, M.J., Zang, Z., Choi, E.Y., Shin, H.K. and Ji, G.E. 2002. Cytoskeleton reorganization andcytokine production of macrophages by Bifidobacterial cells and cell-free extracts. J. Microbiol. Biotechnol. 12(3), 398-405.

Li, N., Russell, W.M., Douglas-Escobar, M., Hauser, N., Lopez, M. and Neu, J. 2009. Live and heatkilled Lactobacillus rhamnosus GG: Effects on proinflammatory and anti-inflammatory cytokines/ chemokines in gastrostomy-fed infant rats. Pediatr. Res. 66, 203-207.

Loh, T.C., Choe, D.W., Foo, H.L., Sazili, A.Q. and Bejo, M.H. 2014. Effects of feeding differentpostbiotic metabolite combinations produced by Lactobacillus plantarum strains on egg quality and production performance, faecal parameters and plasma cholesterol in laying hens. BMC Vet. Res. 10, 149.

Loh, T.C., Chon, S.W., Foo, H.L. and Law, F.L. 2009. Effects on growth performance, faecalmicroflora and plasma cholesterol after supplementation of spray-dried metabolite to post-weaning rats. Czech J. Anim. Sci. 54(1), 10-16.

Loh, T.C., Thanh, N.T., Foo, H.L., Hair-Bejo, M. and Azhar, B.K. 2010. Feeding of different levels of metabolite combinations produced by Lactobacillus plantarum on growth performance, fecal microflora, volatile fatty acids and villi height in broilers. Anim. Sci. J. 81(2), 205-214.

Loh, T.C., Thu, T.V., Foo, H.L. and Bejo, M.H. 2013. Effects of different levels of metabolite combination produced by Lactobacillus plantarum on growth performance, diarrhoea, gut environment and digestibility of post-weaning piglets. J. Appl. Anim. Res. 41, 200-207.

Lopez, M., Li, N., Kataria, J., Russell, M. and Neu, J. 2008. Live and ultraviolet-inactivatedLactobacillus rhamnosus GG decrease flagellin-induced interleukin-8 production in Caco-2 cells. J. Nutr. 138, 2264-2268.

Maldonado Galdeano, C. and Perdigon, G. 2004. Role of viability of probiotic strains in their persistence in the gut and in mucosal immune stimulation. J. Appl. Microbiol. 97, 673-681.

Matsuguchi, T., Takagi, A., Matsuzaki, T., Nagaoka, M., Ishikawa, K., Yokokura, T. and Yasunobu, Y. 2003. Lipoteichoic acids from Lactobacillus strains elicit strong tumor necrosis factor Alpha-inducing activities in macrophages through toll-like receptor 2. Clin. Diagn. Lab. Immunol. 10(2), 259-266.

Montalto, M., Maggiano, N., Ricci, R., Curigliano, V., Santoro, L., Di Nicuolo, F., Vecchio, F.M., Gasbarrini, A. and Gasbarrini, G. 2004. Lactobacillus acidophilus protects tight Sajunctions from aspirin damage in HT-29 cells. Digestion 69, 225-228. 
Oo, K.M., Lwin, A.A., Kyaw, Y.Y., Tun, W.M., Fukada, K., Goshima, A., Shimada, T. and Okada, S. 2016. Safety and long-term effect of the probiotic FK-23 in patients with hepatitis $\mathrm{C}$ virus infection. Biosci. Microbiota Food Health 35(3), 123-128.

Ou, C.C., Ko, J.L. and Lin, M.Y. 2006. Antioxidative effects of intracellular extracts of yoghurtbacteria on lipid peroxidation and intestine 407 cells. J. Food Drug Anal. 14(3), 304-310.

Ouwehand, A.C., Tölkkö, S., Kulmala, J., Salminen, S. and Salminen, E. 2000. Adhesion of inactivated probiotic strains to intestinal mucus. Lett. Appl. Microbiol. 31, 82-86.

Possemiers, S., Pinheiro, I., Verhelst, A., van den Abbeele, P., Maignien, L., Laukens, D., Reeves, S.G., Robinson, L.E., Raas, T., Schneider, Y.J., van de Wiele, T. and Marzorati, M. 2013. A dried yeast fermentate selectively modulates both the luminal and mucosal gut microbiota and protects against inflammation, as studied in an integrated in vitro approach. J. Agric. Food Chem. 61, 9380-9392.

Price, K.L., Totty, H.R., Lee, H.B., Utt, M.D., Fitzner, G.E., Yoon, I., Ponder, M.A. and Escobar, J. 2010. Use of Saccharomyces cerevisiae fermentation product on growth performance and microbiota of weaned pigs during Salmonella infection. J. Anim. Sci. 88, 3896-3908.

Rehman, H.U., Vahjen, W., Awad, W.A. and Zentek, J. 2007. Indigenous bacteria and bacterial metabolic products in the gastrointestinal tract of broiler chickens. Arch. Anim. Nutr. 61(5), 319-335.

Roberfroid, M. 2007. Prebiotics: the concept revisited. J. Nutr. 137, 830S-837S.

Rosyidah, M., Loh, T., Foo, H., Cheng, X. and Bejo, M. 2011. Effect of feeding metabolitesand acidifier on growth performance, faecal characteristics and microflora in broiler chickens. J. Anim. Vet. Adv. 10, 2758-2764.

Rubinelli, P., Roto, S., Kim, S.A., Park, S.H., Pavlidis, H.O., Mcintyre, D. and Ricke, S.C. 2016. Reduction of Salmonella Typhimurium by fermentation metabolites of Diamond V Original XPC in an in vitro anaerobic mixed chicken cecal culture. Front. Vet. Sci. 3(83), 1-7.

Sakai, Y., Tsukahara, T., Bukawa, W., Matsubara, N. and Ushida, K. 2006. Cell preparation of Enterococcus faecalis strain EC-12 prevents vancomycinresistantenterococci colonization in the cecum of newly hatched chicks. Poult. Sci. 85, 273-277.

Schrezenmeir, J. and De Vrese, M. 2001. Probiotics, prebiotics and synbiotics- approachinga definition. Am. J. Clin. Nutr. 73, 361S-364S.

Shang, L., Fukata, M., Thirunarayanan, N., Martin A.P., Arnaboldi, P., Maussang, D., Berin, C., Unkeless, J.C., Mayer, L. and Abreu, M.T. 2008. Toll-like receptor signaling in small intestinal epithelium promotes B-cell recruitment and IgA production in lamina propria. Gastroenterol. 135, 529-538.

Shareef, A.M., Jamel, Z.T. and Yonis, K.M. 2009. Detection of antibiotic residues in stored poultry products. Iraq J. Vet. Sci. 23(1), 45-48.

Shazali, N., Foo, H.L., Loh, T.C., Choe, D.W. and Rahim, R.A. 2014. Prevalence of antibioticresistance in lactic acid bacteria isolated from the faeces of broiler chicken in Malaysia. Gut Pathog. 6, 1.

Sokol, H., Pigneur, B., Watterlot, L., Lakehdari, O., Bermudez-Humaran, L.G., Gratadoux, J.J. and Langella, P. 2008. Faecalibacterium prausnitzil is an anti-inflammatory commensal bacterium identified by gut microbiota analysis of Crohn disease patients. Proc. Natl. Acad. Sci. U. S. A. 105(43), 16731-16736.

Sugahara, H., Yao, R., Odamaki, T. and Xiao, J.Z. 2017. Differences between live and heatkilled Bifidobacteria in the regulation of immune function and the intestinal environment. Benef. Microbes 8, 463-472.

Taverniti, V. and Guglielmetti, S. 2011. The immunomodulatory properties of probiotic microorganisms beyond their viability (ghost probiotics: proposal of paraprobiotic concept). Genes Nutr. 6, 261-274.

Tejada-Simon, M.V. and Pestka, J.J. 1999. Proinflammatory cytokine and nitric oxide induction in murine macrophages by cell wall and cytoplasmic extracts of lactic acid bacteria. J. Food Prot. 62(12), 1435-1444.

Thanh, N.T., Loh, T.C., Foo, H.L., Hair-Bejo, M. and Azhar, B.K. 2009. Effects of feeding metabolite combinations produced by Lactobacillus plantarum on growth performance, faecal microbial population, small intestine villus height and faecal volatile fatty acids in broilers. Br. Poult. Sci. 50, 298-306.

Thu, T.V., Foo, H.L., Loh, T.C. and Bejo, M.H. 2011. Inhibitory activity and organic acid concentrations of metabolite combinations produced by various strains of Lactobacillus plantarum. Afr. J. Biotechnol. 10(8), 1359-1363.

Tiptiri-Kourpeti, A., Spyridopoulou, K., Santarmaki, V., Aindelis, G., Tompoulidou, E., Lamprianidou, E.E., Saxami, G., Ypsilantis, P., Lampri, E.S., Simopoulos, C., Kotsianidis, I., Galanis, A., Kourkoutas, Y., Dimitrellou, D. and Chlichlia, K. 2016. Lactobacillus casei exerts anti-proliferative effects accompanied by apoptotic cell death and up-regulation of TRAIL in colon carcinoma cells. PLoS One 11(2), e0147960.

Tsilingiri, K., Barbosa, T., Penna, G., Caprioli, F., Sonzogni, A., Viale, G. and Rescigno, M. 2012. 
Probiotic and postbiotic activity in health and disease: comparison on a novel polarised ex-vivo organ culture model. Gut 61, 1007-1015.

Vidal, K., Donnet-Hughes, A. and Granato, D. 2002. Lipoteichoic acids from Lactobacillus johnsonii strain La1 and Lactobacillus acidophilus strain La10 antagonize the responsiveness of human intestinal epithelial HT29 cells to lipopolysaccharide and Gram-negative bacteria. Infect. Immun. 70(4), 2057-2064.
Zorzela, L., Ardestani, S.K., McFarland, L.V. and Vohra, S. 2017. Is there a role for modified probiotics as beneficial microbes: a systematic review of the literature. Benef. Microbes 8, 739-754.

Zuidhof, M.J., Schneider, B.L., Carney, V.L., Korver, D.R. and Robinson, F.E. 2014. Growth, effciency, and yield of commercial broilers from 1957, 1978, and 2005. Poult. Sci. 93, 2970-2982. 